\title{
A Hybrid DWT, PCA and SVD based Digital video watermarking Scheme-A Review
}

\author{
K.A. Rajurkar ${ }^{1}$, Prof. S.K. Nanda ${ }^{2}$ \\ Student of M.E., Department of Electronics \& Telecommunication Engineering, P. R. Patil College of Engineering and \\ Technology, Amravati Maharashtra, India ${ }^{1}$ \\ HOD, Department of Electronics \& Telecommunication Engineering, P. R. Patil College of Engineering and \\ Technology, Amravati Maharashtra, India ${ }^{2}$
}

\begin{abstract}
Digital Watermarking is a technology used for the copyright protection of digital applications. Watermarking is a concept of embedding a special symbol, watermark, into an electronic document so that a given piece of copyright information is permanently tied to the data. It is an effective way to protect copyright of multimedia data even after its transmission. In recent years image watermarking techniques are grown-up. So digital video watermarking is the next challenging task and it is becoming a current academic research topic. Digital watermarking is a process of embedding watermark into digital document, where watermark can be some binary data, a small image or a seed value. It can also be any arbitrary or serial number, possession identifier, information about the inventor, date etc, that would be inserted into the original digital products. Digital watermarking refers to embedding watermarks in a multimedia documents and files in order to protect them from illegal copying and identifying manipulations. We proposed a technique is Hybrid DWT, PCA and SVD method for digital video watermarking. In two parts a proposed method of watermarking is divided: watermark embedding and watermark extracting.
\end{abstract}

Keywords: DWT, hybrid, PCA, SVD, PSNR, watermark

\section{INTRODUCTION}

Digital watermark is a pattern of bits inserted into a digital video according to a pre-determined transform and are audio ,video or image that identifies the copyright and more robust than spatial domain techniques since they authenticates information. The goal of watermark disperse the watermark in the spatial domain of the video technique is to embed the secret information seamlessly frame making it difficult to remove the watermark through hidden within into original message, which is robust malicious attacks like rotation, scaling, cropping, and against attacks. The use of digital video applications such as video-conferencing, digital television, digital cinema, distance learning, videophone, and video-on-demand has grown very rapidly over the last few years. Today it is much easier for the digital data owners to transfer multimedia data over the internet, and hence the data could be perfectly duplicated and rapidly redistributed on a large scale. Hence, copyright protection has become more important. Digital watermarking is an efficient way to protect the copyright of multimedia data even after its transmission. Watermarking refers to the process of inserting a hidden structure, called a watermark, into a multimedia data that carries either, the owners information or the receiver of the original data object. Broadcast monitoring, replica control, tracing of transaction, and protection of copyright these are the applications of watermarking. Robustness, invisibility and security are the three most important properties that need to be satisfied for such applications. Video watermarking approaches can be classified into two main categories based on the method of hiding watermark bits in the host video. The two categories are: Transform domain technique and Spatial domain technique. In spatial domain watermarking embedding and detection of watermark are carried out by directly calculating the pixel intensity values of the frame of video. On the other side, Transform domain techniques, alter values of spatial pixel of the host geometrical attacks. The most frequently used transform domain techniques are Discrete Fourier Transform (DFT), the Discrete Cosine Transform (DCT), and the Discrete Wavelet Transform (DWT).

In this paper, we propose an imperceptible and robust video watermarking algorithm based on Discrete Wavelet Transform (DWT) and Singular value decomposition. DWT is more computationally efficient than other transform methods like DFT and DCT. Due to its excellent spatio-frequency localization properties. The DWT is used to identify areas in the host video frame where a watermark can be embedded imperceptibly

\section{LITERATURE SURVEY}

${ }^{[1]}$ Video watermarking using wavelet transform and tensor algebra authors provides robust, hybrid watermarking technique based on high-order tensor singular value decomposition and the discrete wavelet transform (DWT). In this, a simple and computationally inexpensive watermarking methodology for embedding a watermark in the transform domain of video is introduced. This was carried out by modifying the highest singular values of the 3D tensors computed form the four wavelet sub-bands of the video frames..

${ }^{[2]}$ A Joint Encryption/Watermarking System for Verifying the Reliability of Medical Images Proposed a joint encryption/water- marking system for the protection of 
medical images. This system is based on an approach transformed in each step of DWT, where L represents Low which combines a substitutive watermarking algorithm, frequency band, $\mathrm{H}$ represents High frequency band and the quantization index modulation, with an encryption subscript behind them represents the number of layers of algorithm. a stream cipher algorithm (e.g., the RC4) or a transforms. LL sub graph represents the lower resolution block cipher algorithm (e.g., the AES in cipher block estimate of the original video, while mid-frequency and chaining (CBC) mode of operation). A new joint high-frequency details sub graph HL, LH and HH watermarking/encryption system, which guarantees a prior represents horizontal edge, vertical edge and diagonal and a posterior protection of medical images. It merges the edge details .

QIM and a cipher algorithm or a block cipher algorithm. System gives access to two distinct messages in the spatial domain and in the encrypted domain, respectively.

"QR Code Watermarking Algorithm based on Wavelet Transform" [3] is proposed in 2013.Author gives digitally invisible watermark is embedded in a QR code image by means of wavelet transform. In the embedding process, a binary image, logo, is transformed into a corresponding watermark and then embedded into a selected sub band. The experimental results illustrated that, for all the cases considered in this paper is more robustness to attacks and as such it can serve as a viable copyright protection and authentication tool. This paper presented a digital watermarking technique, whereby a binary image is watermarked an embedded in a $\mathrm{QR}$ code image .The experimental results demonstrated that the algorithm can be recover the watermark with an acceptable visual quality. The objective measures such as PSNR and NC are subject to magnitude factor.

In Digital Video Watermarking Using PCA and DWT ${ }^{[4]}$, a comprehensive approach for watermarking digital video is introduced. PCA helps in reducing correlation among the wavelet coefficients obtained from wavelet decomposition of each video frame thereby dispersing the watermark bits into the uncorrelated coefficients. The video frames are first decomposed using DWT and the binary watermark is embedded in the principal components of the low frequency wavelet coefficients. The imperceptible high bit rate watermark embedded is robust against various attacks that can be carried out on the watermarked video, such as filtering, contrast adjustment, noise addition and geometric attacks. In Hybrid DCT- SVD Based Robust Watermarking Scheme for Copyright Protection, A hybrid watermarking scheme exploiting the properties of the Discrete Cosine Transform (DCT) and Singular Value Decomposition (SVD) has been proposed ${ }^{[5]}$. A reference image is being formed from the cover image and then its singular values are modified to hide the secret information in an imperceptible way. The security is further enhanced by the zig zag scrambling of the cover image and gray scale watermarks. The robustness of the methodology against the various image processing attacks has been validated with high Normalized Cross Correlation (NCC) values. Also, the imperceptibility of the watermarked image with the original cover image comes out to be high as indicated by high achievable Peak Signal to Noise Ratio (PSNR) values.

\section{III.PROPOSED METHODOLOGY}

\section{A. Discrete Wavelet Transform}

DWT is a transform based on frequency domain As shown in above figure the distributions of the frequency is

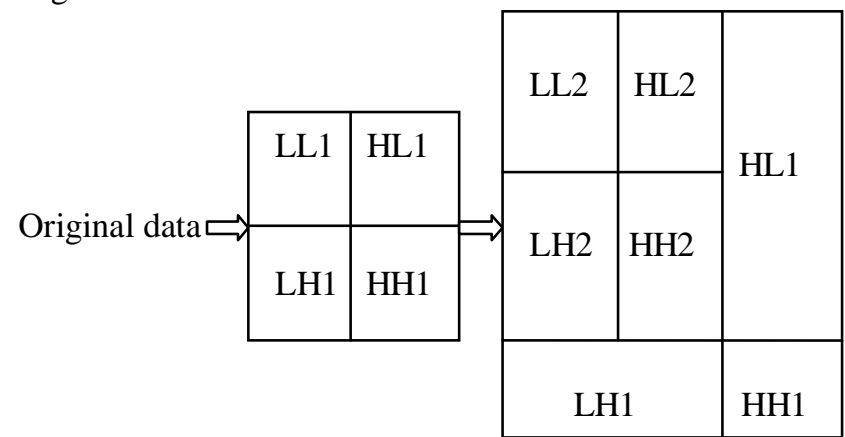

Fig 1: Discrete Wavelet Transform

Above block diagram shows DWT after application of two times. When we apply third level of DWT lower resolution component LL2 again get divide into four sub components.

In proposed watermarking scheme we are using 3-level DWT to increase robustness of the system. After 2 level DWT when we again apply DWT the LL2 component will divide into four sub images as shown above.

\section{B. Singular Value Decomposition}

Singular value decomposition is a numerical technique used to diagonalize matrices in numerical analysis. It is an algorithm developed for a variety of applications. The singular value decomposition (SVD) technique has been successfully used in a variety of applications, such as data compression, pattern analysis and signal processing. It has been scientifically proved that slight variation in the singular values does not change the visual perception of the image. Also, the singular values are robust to the common image processing attacks and do not change much after their application to the image. From the linear algebra viewpoint, the SVD decomposition of any discrete image matrix A of size mxn can be represented as:

$$
\mathrm{A}=\mathrm{USV}^{\mathrm{T}}
$$

Where $\mathrm{U}$ and $\mathrm{V}$ are orthogonal matrices $\left(\mathrm{U}^{\mathrm{T}} \mathrm{U}=\mathrm{I}, \mathrm{V}^{\mathrm{T}} \mathrm{V}=\mathrm{I}\right)$ of size mxm and nxn respectively ${ }^{[5,6]}$. The horizontal and vertical details in an image are given by the columns of $U$ and $\mathrm{V}$ matrices called as left and right singular vectors respectively. The diagonal matrix $S$ with size mxn, has nonzero elements called singular values of the matrix. They represent the luminance values of the image layers and as arranged in decreasing order from the first SV to the last one. The above decomposition is termed as Singular Value Decomposition. The increased robustness is due to the stability of singular values. Singular values exhibit some more properties like rotation invariance, translation invariance, transposition invariance, etc. These all properties of SVD are much desirable in image watermarking. 


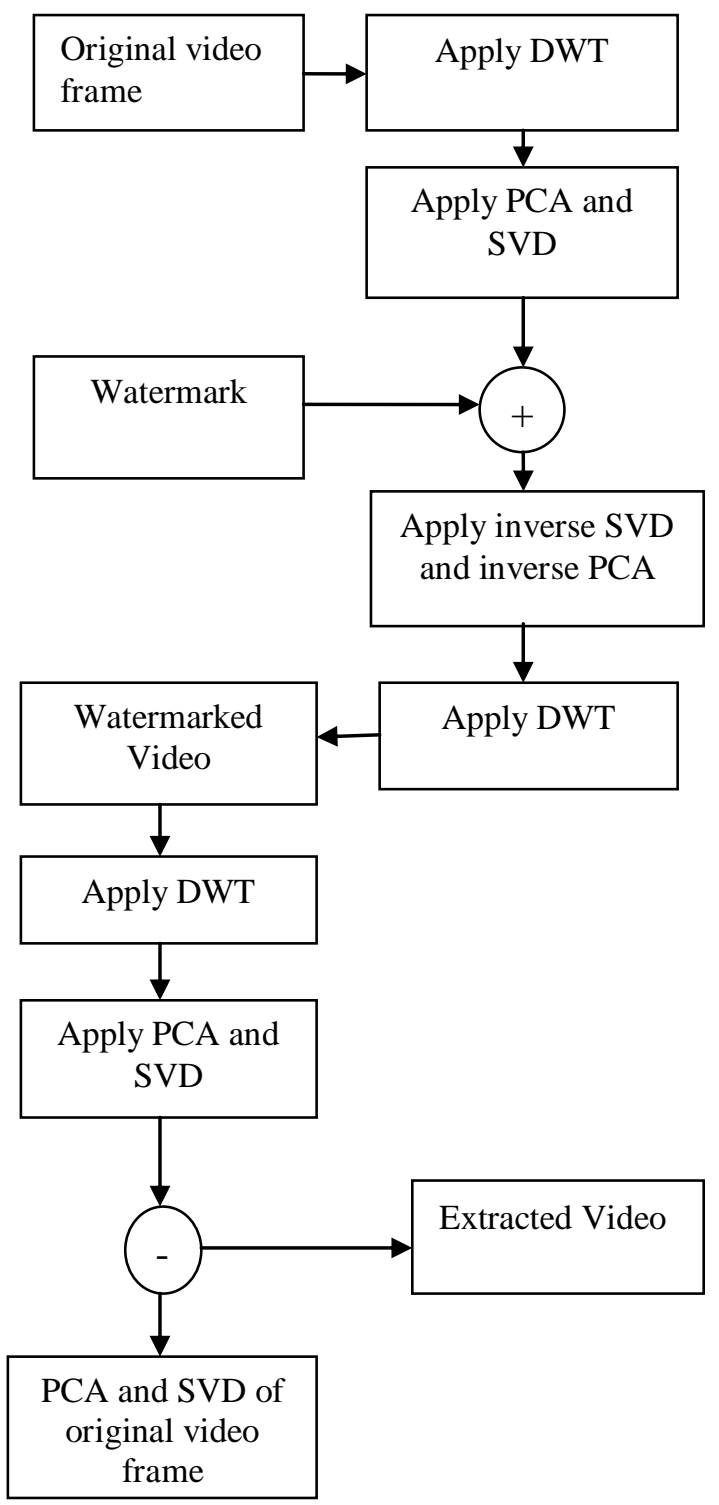

Fig 2: Diagram for Video Watermarking

\section{Principal Component Analysis}

PCA is a mathematical procedure. It converts set of observations of possibly correlated variables into uncorrelated variables by using orthogonal transformation. The number of original variables is greater than or equal to number of principal components. PCA is used to identify patterns in data, and it high lights their similarities and differences. In data of high dimension, where the advantage of graphical representation is not available, patterns in data are hard to find. For analysing data PCA is a powerful tool. The other main benefit of PCA is that once these patterns in the data have been recognized, by reducing the number of dimensions the data can be compressed, without much loss of content. It plots the data with maximum covariance together into a new coordinate system by reducing number of dimensions and is known as the first principal component. Likewise, there are the second and third principal components and so on. first principal component contains maximum energy ${ }^{[7]}$.

\section{Working}

As shown in the block diagram first original video frame get converted into number of frames then frame after particular interval get selected and on that frame DWT is applied which converts image into four sub images. Among them lower resolution approximation sub band image get selected. PCA and SVD is applied on it. After that, watermark is embedded into the processed image as shown in figure.

While extracting image we have to apply DWT on watermarked video followed by PCA and SVD. When we subtract PCA and SVD of original video frame from it .We get extracted video.

\section{CONCLUSION}

When the robustness of the DWT, PCA-SVD based hybrid watermarking scheme will be tested, the performance of the new video watermarking scheme should be evaluated through several experiments. The experiment with various dropping ratio, the various number of frame colluded, the experiment with MPEG compression etc. The NC values will be retrieved when the watermarked video is facing different attacks. The correlation value should be high clearly indicating robustness of the algorithm against major attack. Better performance than the DCT based or SVD based scheme since there are small amount of watermark bits are embedded into frame using DWT, PCA and SVD. It should prevent the attackers from removing the watermark by frame dropping. If they try to remove one part of the watermark, they need to remove more and more frames, leading to a significant damage to the video. In addition, when the frames are dropped; the error is only introduced to a corresponding small part of the watermark. For DWT based scheme, however, the error is introduced to the whole watermark, making the performance worst. We should find that the proposed scheme can resist to statistical averaging quite well. This is because our scheme embedded small amount of watermark into each frame, making the watermarks resistant to attacks by frame averaging for watermark extraction. The NC values of the hybrid approach under lossy compression should be higher than the scheme which only applying single watermarking scheme in a video. There are other attacks like swapping, Salt and Pepper Noise, Sharpen attack, Gaussian Filter Attack, Blurred Attack, Rotation and Rescale Attack etc. The scheme should be robust to fight with all these attacks.

\section{ACKNOWLEDGEMENT}

I would like to express my sincere gratitude to Prof. S. K. Nanda for the necessary support and guidance during various stages of work. I would also like to thank our management for the support because of which we are able to complete our research paper

\section{REFERENCE}

[1] Emad E. Abdallah · A. Ben Hamza · Prabir Bhattacharya, "Video watermarking using wavelet transform and tensor algebra" SIViP April 2009 
[2] Dalel Bouslimi, Gouenou Coatrieux, Michel Cozic, "A JointEncryption/Watermarking System" IEEE, Vol. 16, NO. 5, September 2012.

[3] Jantana Panyavaraporn, Paramate Horkaew, "QR Code Watermarking Algorithm based on Wavelet Transform” ISCIT, 2002

[4] Supriya A. Patil1, Prof. Navin Srivastava "Digital Video Watermarking Using Dwt and PCA"IOSERJEN, vol 3, November 2013M. Shell. (2002)

[5] Priyanka Singh, and Suneeta Agarwal, "A Hybrid DCT- SVD Based Robust Watermarking Scheme for Copyright Protection" ICETET ,2013

[6] Rathod Jigisha , Rachana V.Modi, "A hybrid DWT-SVD method for digital video watermarking" Vol. 2, Issue 7, July 2013

[7] Sanjana Sinha, Prajnat Bardhan, Swarnali Pramanick, Ankul Jagatramka, Dipak K. Kole, Aruna Chakraborty, "Digital Video Watermarking using Discrete Wavelet Transform and Principal component Analysis" International Journal of Wisdom Based Computing, Vol. 1 (2), August 2011

\section{BIOGRAPHIES}

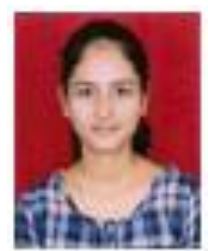

Ms. Kalyani A. Rajurkar received B.E. degree in Electronics and Tele- Communication Engineering from PRMIT \& R, Badnera in year 2013 and currently she is pursuing M.E. in (EXTC) from P.R Maharashtra, India. Patil College of Engineering, Amravati,

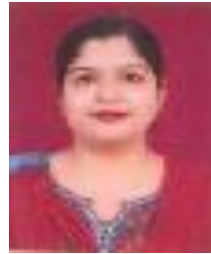

Prof. S. K. Nanda received M. E. degree And is now HOD Department of Electronics and Telecommunication in P. R. Patil College of Engineering, Amravati, Maharashtra, India. Her area of interest is Digital Electronics and has published 4 international and 2 national research papers till now. 